\title{
GRADIENT METHOD FOR NONDENSELY DEFINED CLOSED UNBOUNDED LINEAR OPERATORS
}

\author{
SUNG J. LEE AND M. ZUHAIR NASHED
}

\begin{abstract}
The paper establishes the convergence of the steepest descent method for least-squares solutions of operator equations in Hilbert spaces for any (nondensely defined, unbounded) closed linear operator with closed range. This is done by using a graph topology, an explicit graph topology adjoint, and existing theory of steepest descent for bounded linear operators.
\end{abstract}

1. Introduction. The steepest descent method (gradient method) for bounded linear operators in Hilbert spaces has been extensively studied. See, for example, [5-7, 1416]. This method has also been analyzed via norm topologies for a special class of unbounded operators in $[5,17]$ and for a densely defined regular differential operator in [12].

The purpose of this paper is to show that the steepest descent method can be applied successfully to obtain least-squares solutions of operator equations for any (nondensely defined, unbounded) closed linear operator in Hilbert space (see Theorems 2.2, 3.4 below). This is done by using a graph topology, an explicit graph topology adjoint, and existing theory of steepest descent methods for bounded linear operators. The only assumption we require is that the operator under consideration has a closed range.

We now fix some notation. Let $H_{1}, H_{2}$ be Hilbert spaces and $\langle$,$\rangle be the inner$ product of $H_{1}$ or $H_{2}$. Let $\langle\langle\rangle$,$\rangle be the standard inner product of the direct sum$ $H_{1} \oplus H_{2}$ defined by

$$
\langle\langle\{x, y\},\{z, w\}\rangle\rangle:=\langle x, z\rangle+\langle y, w\rangle
$$

for $\{x, y\},\{z, w\}$ in $H_{1} \oplus H_{2}$. Let $A$ be a linear manifold (subspace) in $H_{1} \oplus H_{2}$. We identify $A$ with the graph of a multi-valued mapping and define

$$
\begin{aligned}
& \text { Dom } A:=\left\{x \in H_{1} \mid\{x, y\} \in A \text { for some } y\right\}, \\
& \text { Range } A:=\left\{y \in H_{2} \mid\{x, y\} \in A \text { for some } x\right\}, \\
& A(x):=\left\{y \in H_{2} \mid\{x, y\} \in A\right\}(x \in \operatorname{Dom} A), \\
& \text { Null } A:=\left\{x \in H_{1} \mid\{x, 0\} \in A\right\}, \\
& A^{-1}:=\{\{x, y\} \mid\{y, x\} \in A\}, \\
& \alpha A:=\{\{x, \alpha y\} \mid\{x, y\} \in A\} \text { for } \alpha \in \mathbf{C} .
\end{aligned}
$$

Received by the editors June 10, 1982 and, in revised form, August 17, 1982.

1980 Mathematics Subject Classification. Primary 47A50, 47A05, 65J10, 65F20.

Key words and phrases. Graph topology, graph topology adjoint, operator part, gradient method, steepest descent, unbounded linear operator, normal equations, iterative methods, generalized inverse of subspace. 
If $V$ is a vector space in $H_{2} \oplus H_{3}$, then $V A:=\left\{\{x, y\} \in H_{1} \oplus H_{3} \mid\{x, z\} \in A\right.$, $\{z, y\} \in V$ for some $z\}$.

For subspaces $A, B$ in $H_{1} \oplus H_{2}$, we define

$$
A+B:=\{a+b \mid a \in A, b \in B\} .
$$

The adjoint (subspace) of $A \subset H_{1} \oplus H_{2}$ is defined by

$$
A^{*}:=\left\{\{y,-x\} \in H_{2} \oplus H_{1} \mid\{x, y\} \in A^{\perp}\right\},
$$

where $A^{\perp}$ denotes the orthogonal complement of $A$.

Important properties of adjoints are $(A \dot{+} B)^{*}=A^{*} \cap B^{*}$ and $(\alpha A)^{*}=\bar{\alpha} A^{*}$. The closure of a set $M$ will be denoted by $M^{c}$.

2. Graph topology adjoint. Let $H_{1}, H_{2}$ be Hilbert spaces and let $M$ be a vector space in $H_{1} \oplus H_{2}$. Assume that $M(0)$ is closed. Let $\mathscr{P}$ denote the graph of the orthogonal projector from $H_{2}$ onto $M(0)$. Define $S_{M}:=(I-\mathcal{P}) M$. Then $S_{M}$ is a linear operator such that Dom $S_{M}=$ Dom $M$, Range $S_{M}=M(0)^{\perp} \cap$ Range $M$, and $M$ is the direct sum of $S_{M}$ and $\{0\} \oplus M(0)$. Note that since $M^{c}$ is closed, so is $S_{M^{c}}$. Also $S_{M^{c}}$ is continuous if and only if Dom $M^{c}$ is closed (see [10]). Define an inner product $\langle,\rangle_{M^{c}}$ on Dom $M$ by

$$
\langle x, y\rangle_{M^{c}}:=\langle x, y\rangle+\left\langle S_{M^{c}}(x), S_{M^{c}}(y)\right\rangle .
$$

If $M^{c}=M$, then Dom $M$ becomes a Hilbert space under this inner product, and the topology generated will be called the graph topology (of $S_{M}$ ) on Dom $M$.

Let $Y$ be the Hilbert space, Dom $M^{c}$ endowed with the graph topology of $S_{M^{c}}$. Let $L \subset Y \oplus \mathrm{H}_{2}$ be a vector space, and let $J$ be the formal operator acting on ordered pairs defined by $J(\{x, y\})=\{y,-x\}$. Then the adjoint, $L_{*}$, of $L$ in $Y \oplus H_{2}$ is defined by

$$
\begin{aligned}
L_{*}=\left\{\{x, y\} \in H_{2} \oplus Y \mid\right. \text { for all } & \{a, b\} \in L, \\
& \left.\langle\langle J(\{a, b\}),\{x, y\}\rangle\rangle=\left\langle S_{M^{c}}(a), S_{M^{c}}(b)\right\rangle\right\} .
\end{aligned}
$$

Note that $L^{*} \subset H_{2} \oplus H_{1}$, whereas $L_{*} \subset H_{2} \oplus$ Dom $M^{c}$.

Let $M \subset H_{1} \oplus H_{2}$ be a vector space such that $M(0)$ is closed. Then

$$
\langle x, u\rangle=0 \text { for all } x \in \operatorname{Dom} M^{*}, u \in M(0) .
$$

Indeed, let $x \in \operatorname{Dom} M^{*}$ and $u \in M(0)$. Then $\{x, z\} \in M^{*}$ for some $z$ and $\{0, u\} \in$ $M$. It follows from definition of $M^{*}$ that

$$
0=\langle\langle J(\{0, u\}),\{x, z\}\rangle\rangle=\langle u, x\rangle .
$$

Since $M(y)=S_{M}(y) \dot{+} M(0)$, it follows from the above property that for any $u \in M(y),\langle x, u\rangle=\left\langle x, S_{M}(y)\right\rangle$. In view of this we define

$$
\langle x, M(y)\rangle:=\left\langle x, S_{M}(y)\right\rangle \text { for all } x \in \operatorname{Dom} M^{*}, y \in \operatorname{Dom} M \text {. }
$$

Proposition 2.1. Let $M \subset H_{1} \oplus H_{2}$ be a vector space such that $M(0)$ is closed. Then $\langle x, M(y)\rangle=\left\langle M^{*}(x), y\right\rangle$ for all $x \in \operatorname{Dom} M^{*}, y \in$ Dom $M$. 
Proof. Take $x \in \operatorname{Dom} M^{*}, \mathrm{y} \in \operatorname{Dom} M$. Then by the above definition $\left\langle M^{*}(x), y\right\rangle=\left\langle S_{M^{*}}(x), y\right\rangle$. Since $\left\{x, S_{M^{*}}(x)\right\} \in M^{*},\left\{y, S_{M}(y)\right\} \in M$, it follows that

$$
0=\left\langle\left\langle J\left(\left\{x, S_{M^{*}}(x)\right\}\right),\left\{y, S_{M}(y)\right\}\right\rangle\right\rangle .
$$

Thus $\left\langle S_{M^{*}}(x), y\right\rangle=\left\langle x, S_{M}(y)\right\rangle=\langle x, M(y)\rangle=\left\langle M^{*}(x), y\right\rangle$.

In the following theorem we characterize the graph topology adjoint via adjoints. It will be convenient to identify a linear operator by its graph.

THEOREM 2.2. Let $M \subset H_{1} \oplus H_{2}$ be a closed linear operator. Then we have the following:

[I] $\left(M S_{M^{*}}+I\right)^{-1} \subset H_{2} \oplus H_{1}$ is an operator.

[II] Let $Y$ be the Hilbert space, Dom $M$, endowed with the graph topology of $M$. Let $(M)_{*} \subset H_{2} \oplus Y$ be the adjoint of $M$ taken in $Y \oplus H_{2}$. Then

(i) Dom $M_{*}=H_{2}$; Range $M_{*} \subset \operatorname{Dom} M ; M_{*}=S_{M^{*}}\left(M S_{M^{*}}+I\right)^{-1} ; M_{*}$ is a continuous operator on $\mathrm{H}_{2}$ into $\mathrm{Y}$.

(ii) Null $M_{*}=$ Null $M^{*}=$ Null $M M^{*} ; \operatorname{Range} M_{*}=($ Dom $M) \cap\left(\operatorname{Range} M^{*}\right)$. Moreover, the following statements are equivalent:

(a) Range $M_{*}$ is closed in $Y$.

(b) Range $M$ is closed in $\mathrm{H}_{2}$.

(c) Range $M^{*}$ is closed in $H_{1}$.

Proof. [I] Take $x \in \operatorname{Dom} M S_{M^{*}}$. Then $x \in \operatorname{Dom} M^{*}$ and $S_{M^{*}}(x) \in \operatorname{Dom} M$. Thus by Proposition 2.1, $\left\langle M S_{M^{*}}(x), x\right\rangle=\left\|S_{M^{*}}(x)\right\|^{2}$. Suppose further that $M S_{M^{*}}(x)+x=0$. Then

$$
\begin{aligned}
0 & =\left\langle M S_{M^{*}}(x)+x, M S_{M^{*}}(x)+x\right\rangle \\
& =\left\|M S_{M^{*}}(x)\right\|^{2}+\|x\|^{2}+2\left\|S_{M^{*}}(x)\right\|^{2} .
\end{aligned}
$$

Thus $x=0$, and so $M S_{M^{*}}+I$ is one-to-one.

[II] Since $M$ is continuous on $Y$ into $H_{2}, M_{*}$ is also continuous on $H_{2}$ into $Y$ (with the same graph norms). We show first that

$$
L:=S_{M^{*}}\left(M S_{M^{*}}+I\right)^{-1} \subset M_{*} \text {. }
$$

Take $\{x, y\} \in L$. Put $g:=\left(M S_{M^{*}}+I\right)^{-1}(x)$. Then $\{g, y\} \in S_{M^{*}} \subset M^{*}$. Take any $\{a, b\} \in M$. Then

$$
\begin{aligned}
\langle\langle J(\{a, b\}),\{x, y\}\rangle\rangle & =\left\langle\left\langle J(\{a, b\}),\left\{\left(M S_{M^{*}}+I\right)(g), y\right\}\right\rangle\right\rangle \\
& =\left\langle\left\langle J(\{a, b\}),\left\{M S_{M^{*}}(g), 0\right\}\right\rangle\right\rangle+\langle\langle J(\{a, b\}),\{g, y\}\rangle\rangle \\
& =\left\langle b, M S_{M^{*}}(g)\right\rangle=\langle b, M(y)\rangle .
\end{aligned}
$$

It follows that $\{x, y\} \in M_{*}$, and so $L \subset M_{*}$. Thus to show that $L=M_{*}$ it suffices to show that Dom $L=H_{2}$. Note that $\operatorname{Dom} L=\operatorname{Range}\left(M S_{M^{*}}+I\right)$, where $S_{M^{*}}=$ $(I-Q) M^{*}$, by definition, and $Q$ is the graph of the orthogonal projector of $\mathrm{H}_{2}$ onto Null $M^{*}$. Using Proposition 3.52 of [1], we obtain

$$
I+M S_{M^{*}}=I+M(I-Q)(I-Q) M^{*}=I+\left[(I-Q) M^{*}\right]^{*}(I-Q) M^{*} .
$$


Put $T:=(I-Q) M^{*}$. Then $I+M S_{M^{*}}=I+T^{*} T$. Since $M$ is closed, so is $T$ (see [10]). It then follows that $H_{2} \oplus H_{2}=T \dot{+} T^{\perp}$. Thus

$$
H_{2} \oplus H_{2}=J\left(T \dot{+} T^{\perp}\right)=J(T) \dot{+} T^{*} .
$$

Let $y \in H_{2}$. Then $\{0,-y\}=J(\{x, T(x)\})+\left\{s, T^{*}(s)\right\}$ for some $x \in \operatorname{Dom} T, s \in$ Dom $T^{*}$. It follows that $x \in \operatorname{Dom} T^{*} T$ and $y=x+T^{*} T x$. This shows that Dom $L$ $=\operatorname{Range}\left(T^{*} T+I\right)=H_{2}$, and so $L=M_{*}$. This proves (i). It follows immediately from $L=M_{*}$ that Range $M_{*}=($ Dom $M) \cap\left(\right.$ Range $\left.M^{*}\right)$.

We will show that Null $M^{*}=\operatorname{Null} M_{*}$. Note first that $\langle\langle J(\{a, b\}),\{x, y\}\rangle\rangle=$ $\langle b, M(y)\rangle$ for all $\{a, b\} \in M,\{x, y\} \in M_{*}$. It follows that $\{x, 0\} \in M_{*}$ if and only if $x \in H_{2}$ and $\langle b, x\rangle=0$ for all $b \in \operatorname{Range} M$. Thus Null $M_{*}=(\operatorname{Range} M)^{\perp}=$ Null $M^{*}$ as $M$ is assumed to be closed, and it is easy to show that Null $M M^{*}=$ Null $M^{*}$. The last part of (ii) follows from Theorem 2.3 of [2].

REMARKs. (i) Dom $M^{*}$ is dense in $H_{2}$ if and only if $M^{c}$ is an operator [1, 2].

(ii) If $M^{*}$ is an operator (or equivalently, Dom $M$ is dense in $H_{1}$ ), then $M^{*}=S_{M^{*}}$.

(iii) In the case when $M$ is the graph of a densely defined closed linear operator generated by an ordinary differential expression, it was shown in [12] that $M_{*}=$ $M^{*}\left(M M^{*}+I\right)^{-1}$. However, the proof there is not complete as it was not shown that Dom $M^{*}\left(M M^{*}+I\right)^{-1}=L_{2}[a, b]$.

(iv) In the case when $M$ is the graph of an ordinary differential operator, the convergence in the graph topology implies uniform convergence. Thus $M_{*}$ is useful in studying $L$-splines discussed in $[4,13]$.

(v) The usefulness of Theorem 2.2 depends on explicit characterization of $M^{*}$. Basically, there are two notable methods for this. One is developed by E. A. Coddington, which makes heavy use of subspaces (see [2] and related references cited therein), and the other method utilizes boundary operators and Green's formulas (see [9]).

3. Steepest descent method in graph topology. In this section, we will give a satisfactory treatment of the steepest descent method for nondensely defined unbounded linear operators. The only restriction will be that their ranges are closed. The main idea is that any closed operator becomes a bounded operator in graph topology, and hence an existing theory for bounded operators (such as that in [15]) is applicable. This is made possible by the explicit characterization of graph topology adjoints derived in Theorem 2.2 .

Suppose that $M \subset H_{1} \oplus H_{2}$ is a vector space and $g \in H_{2}$. Then $u \in H_{1}$ is called a least-sequence solution of the relation $g \in M(x)$ (or equivalently $\{x, g\} \in M$ ) if (i) $u \in \operatorname{Dom} M$, (ii) $d(g$, Range $M)=\|g-s\|$ for some $s \in M(u)$.

The following was proved in [11].

THEOREM 3.1. Let $M \subset H_{1} \oplus H_{2}$ be a closed vector space and $g \in H_{2}$. Then we have the following:

[I] $u \in H_{1}$ is a least-squares solution of $g \in M(x)$ if and only if $u \in \operatorname{Dom} M$ and $g \in M(u)+$ Null $M^{*}$. Moreover, $g \in M(x)$ has a least-squares solution if and only if $g \in$ Range $M+$ Null $M^{*}$. 
[II] Assume that $g \in$ Range $M+$ Null $M^{*}$. Let $M^{\#}$ denote the orthogonal generalized inverse (the analogue of the Moore-Penrose generalized inverse) of the subspace $M$, that is $M^{\#}:=(I-\mathcal{P}) M^{-1}\left(I-\mathcal{P}^{+}\right)$, where $\mathcal{P}$ and $\mathcal{P}^{+}$are the graphs of the orthogonal projectors from $H_{1}$ and $H_{2}$ onto Null $M$ and Null $M^{*}$, respectively. Then:

(i) $M^{\#}(g)+$ Null $M$ is the set of all least-squares solutions of $g \in M(x)$.

(ii) $\left\|M^{\#}(g)\right\| \leqslant\|u\|$ for all least-squares solutions $u$ of $g \in M(x)$. Moreover, whenever $u$ is a least-squares solution of $g \in M(x)$ with $\|u\| \leqslant\left\|M^{\#}(g)\right\|$, then $u=M^{\#}(g)$.

In the rest of this section we will compute least-squares solutions of a nondensely defined closed linear operator by a steepest descent method. The topology used will be a graph topology, and a graph topology adjoint will play a fundamental role. The gradient method for $K$-positive definite unbounded operators was investigated in [5 and 17] but our approach is more natural and general.

The steepest descent method in graph topology for least-squares solutions of regular ordinary differential operators was first investigated by Locker [12]. Our abstract approach has been motivated by the explicit characterization of the graph topology adjoint in [12].

Proposition 3.2. Let $M \subset H_{1} \oplus H_{2}$ be a closed linear operator. Then $u \in H_{1}$ is a least-squares solution of $g=M(x)$ if and only if $u \in \operatorname{Dom} M$ and $M_{*}(M(u)-g)=0$.

Proof. By Theorem 3.1, $u \in H_{1}$ is a least-squares solution of $g=M(x)$ if and only if $u \in \operatorname{Dom} M$ and $g \in M(u)+$ Null $M^{*}$. By Theorem 2.2, Null $M^{*}=$ Null $M_{*}$. It follows that $g \in M(u)+$ Null $M^{*}$ if and only if $M_{*}(M(u)-g)=0$.

The following proposition gives a connection between the orthogonal generalized inverse of $M$ in a norm topology and the one in a graph topology.

Proposition 3.3. Let $M \subset H_{1} \oplus H_{2}$ be a closed linear operator. Let $M_{\#}$ denote the orthogonal generalized inverse of $M$ taken in $Y \oplus H_{2}$, where $Y$ is the Hilbert space, Dom $M$, equipped with the graph topology of $M$. Then $M_{\#}=M^{\#}$. In particular,

$$
\begin{aligned}
\text { Dom } M_{\#} & =\text { Range } M+\operatorname{Null} M^{*}, \\
\text { Range } M_{\#} & =(\operatorname{Dom} M) \cap(\operatorname{Null} M)^{\perp} .
\end{aligned}
$$

Proof. Let $Y$ be the Hilbert space, Dom $M$, endowed with the graph topology of $M$. Let $\mathcal{P}$ be the orthogonal projector from $H_{1}$ onto Null $M$. Now

$$
(\text { Null } M)_{\perp}=(\operatorname{Dom} M) \cap(\operatorname{Null} M)^{\perp}
$$

where for $U \subset Y, U_{\perp}$ denotes the orthogonal complement of $U$ in $Y$. Thus $\mathscr{P}_{0}:=\left.\mathscr{P}\right|_{\operatorname{Dom} M}$ is the orthogonal projector from $Y$ onto Null $M$. Let $\mathcal{P}^{+}$be the orthogonal projector from $\mathrm{H}_{2}$ onto Null $M^{*}$. Since Null $M^{*}=\operatorname{Null} M_{*}$, it follows that $\mathscr{P}^{+}$is also the orthogonal projector from $H_{2}$ onto Null $M_{*}$. Since

$$
M^{\#}:=(I-\mathcal{P}) M^{-1}\left(I-\mathcal{P}^{+}\right), \quad M_{\#}:=\left(I-\mathcal{P}_{0}\right) M^{-1}\left(I-\mathcal{P}^{+}\right),
$$

it follows that $M^{\#}=M_{\#}$ as it is clear that $\left(I-\mathscr{P}_{0}\right) M^{-1}=(I-\mathscr{P}) M^{-1}$. The last part was proved in [10]. 
The following is the main theorem of this section.

THEOREM 3.4. Let $M \subset H_{1} \oplus H_{2}$ be a closed linear operator. Let $g \in H_{2}$ and $x_{0} \in$ Dom $M$ be given. Define a sequence $\left\{x_{n}\right\}$ recursively as follows:

$$
x_{n+1}=x_{n}-\alpha_{n} r_{n} \quad(n=0,1,2, \ldots),
$$

where $\alpha_{n} \geqslant 0$ and $r_{n} \in$ Dom $M$ are defined by

$$
\left\{\begin{array}{l}
r_{n}=S_{M^{*}}\left(M S_{M^{*}}+I\right)^{-1}\left(M\left(x_{n}\right)-g\right) \\
\alpha_{n}\left\|M\left(r_{n}\right)\right\|^{2}=\left\|r_{n}\right\|^{2}+\left\|M\left(r_{n}\right)\right\|^{2}
\end{array}\right.
$$

Then we have the following:

(i) $r_{n}=0$ if and only if $M\left(r_{n}\right)=0$, or equivalently, $x_{n}$ is a least-squares solution of $g=M(x)$.

(ii) Assume that $r_{n} \neq 0$ for all $n=0,1,2, \ldots$ Then

$$
x_{n+1}=x_{0}-\sum_{i}^{n} \frac{\left\|r_{i}\right\|^{2}+\left\|M\left(r_{i}\right)\right\|^{2}}{\left\|M\left(r_{i}\right)\right\|^{2}} r_{i} \quad(n=0,1,2, \ldots),
$$

and $\left\{r_{n}\right\}$ converges to the zero vector in the graph topology in Dom $M$.

(iii) Assume $r_{n} \neq 0$ for all $n=0,1,2, \ldots$, and Range $M$ is closed in $H_{2}$. Then $\left\{x_{n}\right\}$ converges in graph topology of $M$ to a least-squares solution of $g=M(x)$ and to $M^{\#}(g)$ if and only if $x_{0} \in$ Range $M^{*}$.

Proof. Let $Y$ be the Hilbert space, Dom $M$, endowed with the graph topology of $M$. Let $M_{*}$ be the adjoint of $M$ taken in $Y \oplus H_{2}$. Then by Theorem 2.2, $M_{*}=$ $S_{M^{*}}\left(M S_{M^{*}}+I\right)^{-1}$. The norm $\|x\|_{M}$ in $Y$ is given by $\left(\|x\|^{2}+\|M(x)\|^{2}\right)^{1 / 2}$. Now for $g \in H_{2}, u$ is a least-squares solution of $g=M(x)$ if and only if $u \in$ Dom $M$ and $M_{*}(M(u)-g)=0$ (Proposition 3.2), and by Theorem 3.1 and Proposition 3.3, $M^{\#}(g)=M_{\#}(g)$ is a least-squares solution of $g=M(x)$, whenever $g \in$ Range $M \dot{+}$ Null $M^{*}$. Thus the result follows from the argument used in [15] (see also Theorem 3.1 of [5]).

REMARK. Since $M$ is a bounded linear operator on $Y$ into $H_{2}$, the convergence rates of $\left\{x_{n}\right\}$ can be discussed as in $[\mathbf{1 4}, 5]$ where the notation $T$ is used instead of $M$.

REMARK. In the case when $M$ is the graph of a nondensely defined differential operator with finitely or infinitely many boundary conditions, Theorem 3.4 can be made more explicit by characterizing $M^{*}$ and $S_{M^{*}}$ using the results of [9 or 2].

REMARK. The results of this paper constitute a final chapter in the theory of the steepest descent iterative method for (least-squares) solutions of closed linear operator equations in Hilbert space. By establishing convergence of this method for nondensely defined unbounded linear operators we have shown a universality property of the method, freeing the convergence theory from conditions that have been imposed by earlier authors (ranging from positive definite bounded operators as in the work of G. Temple and L. V. Kantorovich, to bounded or certain closed densely defined operators as in the work of several authors mentioned earlier in this paper). A crucial use is made of viewing the operator in terms of its graph and of an 
explicit graph topology adjoint. Certain advantages of studying single-valued operators in terms of their graphs were considered in a seminal paper by Hestenes [3]. The techniques of the present paper can be easily adapted to extend other iterative methods (e.g. $[8,14,16,18])$ to nondensely defined closed linear operators in Hilbert space. Notice that if $M$ is a closable linear operator, then in Theorem 2.2, Propositions 3.2, 3.3 and Theorem 3.4 one can repalce $M$ by $M^{c}$, the closure of $M$.

\section{REFERENCES}

1. R. Arens, Operational calculus of linear relations, Pacific J. Math. 11 (1961), 9-23.

2. E. A. Coddington and A. Dijksma, Adjoint subspaces in Banach spaces, with application to ordinary differential subspaces, Ann. Mat. Pura. Appl. 118 (1978), 1-118.

3: M. R. Hestenes, Relative self-adjoint operators in Hilbert spaces, Pacific J. Math. 11 (1961), 1315-1357.

4. J. W. Jerome and L. L. Schumaker, On Lg-splines, J. Approx. Theory 2 (1969), 29-49.

5. W. J. Kammerer and M. Z. Nashed, Steepest descent for singular linear operators with nonclosed range, Applicable Anal. 1 (1971), 143-159.

6. Iterative methods for hest approximate solutions of linear integral equations of the first and second kinds, J. Math. Anal. Appl. 40 (1972), 547-573.

7. L. V. Kantorovich and G. P. Akilov, Functional analysis in normed spaces, Pergamon Press, London and New York, 1964.

8. L. J. Lardy, A series representation for generalized inverse of a closed linear operator. Atti Acad. Naz. Lincei Rend. Cl. Sci. Fis. Mat. Natur. 58 (1975), 152-157.

9. S. J. Lee, Boundary conditions for linear manifolds, I, J. Math. Anal. Appl. 73 (1980), 366-380.

10. S. J. Lee and M. Z. Nashed, Operator parts and generalized inverses of multi-valued operators, with applications to ordinary differential subspaces (to appear).

11. Least-squares solutions of multi-valued linear operators, J. Approx. Theory (to appear).

12. J. Locker, Weak steepest descent for linear houndary value problems, Indiana Univ. Math. J. 25 (1976), 525-530.

13. T. R. Lucas, $A$ generalization of L-splines, Numer. Math. 15 (1970), 359-370.

14. S. F. McCormick and G. H. Rodrigue, A uniform approach to gradient methods for linear operator equations, J. Math. Anal. Appl. 49 (1975), 275-285.

15. M. Z. Nashed, Steepest descent for singular linear operator equations, SIAM J. Numer. Anal. 7 (1970), 358-362.

16. Perturbations and approximations for generalized inverses and linear operator equations, Generalized Inverses and Applications (M. Z. Nashed, ed.), Academic Press, New York, 1976, pp. 325-396.

17. W. V. Petryshyn, Direct and iterative methods for the solution of linear operator equations in Hilbert space, Trans. Amer. Math. Soc. 105 (1962), 136-175.

18. On generalized inverses and uniform convergence of $(I-\beta K)^{n}$ with applications to iterative methods, J. Math. Anal. Appl. 18 (1967), 417-439.

Department of Mathematics, University of South Florida, Tampa, Florida 33620

Department of Mathematical Sciences, University of Delaware, Newark, Delaware 19711 